Pacific Journal of Mathematics

BOUNDARY BEHAVIOR OF A CONFORMAL MAPPING 


\section{BOUNDARY BEHAVIOR OF A CONFORMAL MAPPING}

\section{JoHn MARAFINo}

Let $D$ be a simply connected plane domain, not the whole plane. Let $R^{*}$ denote those accessible boundary points of $D$ such that $D$ twists violently about them; that is, if $\alpha \in R^{*}$ and $w(\alpha)$ denotes its complex coordinate, then

$$
\begin{aligned}
& \lim _{\substack{w \rightarrow \alpha \\
w \in D}} \inf \arg (w-w(\alpha))=-\infty \text { and } \\
& \lim _{\substack{w \rightarrow \alpha \\
w \in D}} \sup \arg (w-w(\alpha))=+\infty,
\end{aligned}
$$

where $\arg (w-w(\alpha))$ is defined and continuous in $D$. We show that if a certain geometric condition holds at each point of a set $W^{*} \subset R^{*}$, then $W^{*}$ is a $D$-conformal null set. Let $L_{\nu}$ denote the ray with terminal point $w(\alpha), \alpha \in R^{*}$, having inclination $\nu, 0 \leq \nu<2 \pi$. Let $m$ denote Lebesgue measure on $L_{\nu}$ and set

$$
u(\nu)=\limsup _{r \rightarrow 0} \frac{m\left(\left(L_{\nu} \cap D\right) \cap\left(w(\alpha), w(\alpha)+r e^{i \nu}\right)\right)}{r} .
$$

Let $W^{*}=\left\{\alpha \in R^{*}\right.$ : there exists $L_{\nu_{i}}, i=1,2,3$, at $w(\alpha)$ such that $\left|\nu_{i}-\nu_{j}\right|=(2 / 3) \pi, 1 \leq i<j \leq 3$, and $u\left(\nu_{i}\right)<1$ for $i=$ $1,2,3\}$.

THEOREM. $W^{*}$ is a D-conformal null set.

Introduction. Let $D$ be a simply connected plane domain, not the whole plane, and let $w=f(z)$ be a one to one conformal map from the open unit disk onto $D$. It is well-known that for almost every $\theta, 0 \leq \theta<2 \pi, f(z)$ has a finite radial limit $f\left(e^{i \theta}\right)$ at $e^{i \theta}$. By [4, pp. 311-312] we also have for almost every $\theta$ that the image of the radius at $e^{i \theta}$ is a rectifiable curve. Thus, for almost every $\theta$, $0 \leq \theta<2 \pi$, the image of the radius at $e^{i \theta}$ determines a (ideal) rectifiably accessible boundary point $\alpha_{\theta}$ of $D$ whose complex coordinate $w\left(\alpha_{\theta}\right)=f\left(e^{i \theta}\right)$ is finite. The set of all such $\alpha_{\theta}$ is denoted by $A^{*}$. In fact, using Theorem 1 in [2, p. 37], Theorem 9.3 in [4, p. 268], and Theorem 10.9 in [4, p. 316], it follows that $A^{*}$ is the set of all rectifiably accessible boundary points of $D$. On $D^{*}=D \cup A^{*}$ we define the arc-length distance $l_{D^{*}}$ between two points as the infimum of the euclidean lengths of arcs that lie in $D$ and join these two points. 
It can be shown that $l_{D^{*}}$ is a metric for $D^{*}$. Any limits involving accessible boundary points are taken in $l_{D^{*}}$.

A set $N^{*} \subset A^{*}$ is said to be a $D$-conformal null set provided that the set $\left\{\theta: \alpha_{\theta} \in N^{*}\right\}$ has measure zero. We note that this definition is independent of $f$. We shall let $T^{*}$ be those $\alpha \in A^{*}$ at which the inner tangent to the boundary of $D$ exists. Thus, if $\alpha \in T^{*}$ then there exists a unique $\nu(\alpha), 0 \leq \nu(\alpha)<2 \pi$, such that for each $\varepsilon>0$ $(\varepsilon<\pi / 2)$ there exists a $\delta>0$ such that

$$
\Delta=\left\{w(\alpha)+\rho e^{i \nu}: 0<\rho<\delta,|\nu-\nu(\alpha)|<\pi / 2-\varepsilon\right\} \subset D
$$

and $w \rightarrow \alpha$ as $w \rightarrow w(\alpha), w \in \Delta$. We denote by $R^{*}$ the set of rectifiably accessible boundary points of $D$ such that

$\liminf _{\substack{w \rightarrow \alpha_{\theta} \\ w \in D}} \arg \left(w-w\left(\alpha_{\theta}\right)\right)=-\infty$ and $\limsup _{\substack{w \rightarrow \alpha_{\theta} \\ w \in D}} \arg \left(w-w\left(\alpha_{\theta}\right)\right)=+\infty$

where $\arg \left(w-w\left(\alpha_{\theta}\right)\right)$ is defined and continuous in $D$.

In [3, p. 44] a diameter metric is used and it is shown that $A^{*}=$ $T^{*} \cup R^{*} \cup N^{*}$, where $N^{*}$ is a $D$-conformal null set. Also, an example is given [3, p. 65] of a domain $D$ where $A^{*}=R^{*} \cup N^{*}$. We note that the same characterization of $A^{*}$ holds using $l_{D^{*}}$ and that in the cited example each point of $A^{*}$ is rectifiably accessible.

In this paper we shall restrict our attention to $R^{*}$ and show that if a certain geometric condition holds at each point of a set $W^{*} \subset R^{*}$, then $W^{*}$ is a $D$-conformal null set. Let $L_{\nu}$ denote the ray with terminal point $w(\alpha), \alpha \in R^{*}$, having inclination $\nu, 0 \leq \nu<2 \pi$. Let $m$ denote Lebesgue measure on $L_{\nu}$ and set

$$
u(\nu)=\limsup _{r \rightarrow 0} \frac{m\left(\left(L_{\nu} \cap D\right) \cap\left(w(\alpha), w(\alpha)+r e^{i \nu}\right)\right)}{r} .
$$

Let $W^{*}=\left\{\alpha \in R^{*}\right.$ : there exists $L_{\nu_{i}}, i=1,2,3$, at $w(\alpha)$ such that $\left|\nu_{i}-\nu_{j}\right|=(2 / 3) \pi, 1 \leq i<j \leq 3$, and $u\left(\nu_{i}\right)<1$ for $\left.i=1,2,3\right\}$.

THEOREM. $W^{*}$ is a D-conformal null set.

The proof of this theorem will be a consequence of the lemma stated below. Given $\alpha \in R^{*}$ and positive numbers $\nu, \delta^{\prime}, \delta^{\prime \prime}, \varepsilon$ with $\delta^{\prime}<\delta^{\prime \prime}$ and $\varepsilon<\pi / 2$, we define $A\left(\alpha, \nu, \delta^{\prime}, \delta^{\prime \prime}, \pi / 2-\varepsilon\right)=\left\{w(\alpha)+\rho e^{i \phi}\right.$ : $\left.|\phi-\nu|<\pi / 2-\varepsilon, \delta^{\prime}<\rho<\delta^{\prime \prime}\right\}$.

LEMMA. Except for a $D$-conformal null set of $R^{*}$, for each $\alpha \in R^{*}$ there exists a $\nu(\alpha), 0 \leq \nu(\alpha) \leq 2 \pi$, and a sequence of pairwise disjoint 
disks $\left\{O_{n}\right\}$ with radii $r_{n}$ and center $w_{n}$ such that for each $\varepsilon>0$, $\varepsilon<\pi / 2$, there exists sequences $\left\{\delta_{n}^{\prime}\right\},\left\{\delta_{n}^{\prime \prime}\right\}$ of positive real numbers such that large $n$.

(1) $A\left(\alpha, \nu(\alpha), \delta_{n}^{\prime}, \delta_{n}^{\prime \prime}, \pi / 2-\varepsilon\right) \subset O_{n} \subset D$ for all sufficiently

(2) $l_{D^{*}}\left(w_{n}, \alpha\right) \rightarrow 0$ as $n \rightarrow \infty$. Consequently, $r_{n}, \delta_{n}^{\prime}, \delta_{n}^{\prime \prime} \rightarrow 0$ as $n \rightarrow \infty$.

(3)

$$
\lim _{n \rightarrow \infty} \frac{\delta_{n}^{\prime \prime}-\delta_{n}^{\prime}}{r_{n}}=2 \cos (\pi / 2-\varepsilon) \text { and } \lim _{n \rightarrow \infty} \frac{\delta_{n}^{\prime \prime}-\delta_{n}^{\prime}}{\delta_{n}^{\prime \prime}}=1 .
$$

Note that as we approach $\alpha \in T^{*}$ through the regions $\Delta$, the measure of the angle at the vertex approaches $\pi$. Because of this property our theorem is trivially true on $T^{*}$. Our lemma on $R^{*}$ asserts that the regions $A\left(\alpha, \nu(\alpha), \delta_{n}^{\prime}, \delta_{n}^{\prime \prime}, \pi / 2-\varepsilon\right)$ are mimicking this property of the $\Delta$ 's and as a consequence our theorem should hold on $R^{*}$.

Proof of Lemma. Our lemma follows from a result due to Gerasch [1] which we shall indicate in our discussion below.

Let $\eta_{k}$ and $\delta_{k}, k=1,2, \ldots$, be sequences of positive numbers decreasing to zero. For any sequence $\left\{w_{n}\right\}$ of points in $D$ we define the corresponding sequence of disks $\left\{O_{n}\right\}$ as follows: $O_{n}$ is the largest disk centered at $w_{n}$ having radius $r_{n}$ that is contained in $D$. We will say that a sequence $\left\{w_{n}\right\} \subset D$ is a boundary sequence provided that the Euclidean distance from $w_{n}$ to the boundary of $D$ approaches zero as $n \rightarrow \infty$. We let $F_{k, p}^{*}$ be those points $\alpha \in R^{*}$ such that for all boundary sequences $\left\{w_{n}\right\}$ the corresponding sequence of disks $\left\{O_{n}\right\}$ satisfy $l_{D^{*}}\left(O_{n}, \alpha\right)>\eta_{k} r_{n}, r_{n}<\delta_{p}$. Set $F_{k}^{*}=\bigcup_{p} F_{k, p}^{*}$.

In [1, p. 204] Gerasch uses an extremal length argument to show that a set, whose definition is similar to $F_{k, p}^{*}$, is $D$-conformal null. The steps of his proof can be used here to show $F_{k, p}^{*}$ is $D$-conformal null. In the following three paragraphs we outline this procedure. For more details we refer the reader to Gerasch's paper. We also note that the extremal length argument used by Gerasch was also used by McMillan in [3, pp. 58-61] to establish his characterization of $A^{*}$.

Let $E=\left\{e^{i \theta}: \alpha_{\theta} \in F_{k, p}^{*}\right\}$. It is well known that almost every point of a set of positive measure is a point of outer density. If we show that each point $e^{i \theta} \in E$ is not a point of outer density then it follows that $m(E)=0$. In order to do this we will first establish a relationship between the extremal length of a family of curves in $\{|z|<1\}$ and 
the density of $E$ near $e^{i \theta} \in E$. We use the following result which is due to Gerasch [1, p. 202]; McMillan proves a similar result on the upper half plane [3, p. 56]:

Let $0<\delta<\pi / 2$ and let $A \subset(0, \delta)$ with outer measure $m^{*}(A)$. For each $\theta$ such that $0<\theta<\delta$, let $\gamma_{\theta}$ denote the arc of the circle orthogonal to $|z|=1$ at $e^{i \theta}$ and at $e^{-i \theta}$ which is contained in the unit disk. Set $\Gamma=$ $\left\{\gamma_{\theta}: \theta \in A\right\}$. Then the extremal length $\lambda(\Gamma)$ of the family of curves satisfies

$$
\lambda(\Gamma) \leq \pi / \log k,
$$

where $k=\sin \delta / \sin \left[\delta-m^{*}(A)\right]$.

Fix $e^{i \theta_{0}} \in E$ and let $\left\{z_{n}\right\}$ be a radial sequence approaching $e^{i \theta_{0}}$. The sequence $\left\{w_{n}\right\}, w_{n}=f\left(z_{n}\right)$, satisfies $l_{D^{*}}\left(w_{n}, \alpha_{\theta_{0}}\right) \rightarrow 0$ because $f$ has radial limit $\alpha_{\theta_{0}}$, and so $r_{n} \rightarrow 0$ as $n \rightarrow \infty$. Thus $\left\{w_{n}\right\}$ is a boundary sequence whose corresponding sequence of disks $\left\{O_{n}\right\}$ satisfy

$$
\text { For all } \theta \in E, \quad l_{D^{*}}\left(O_{n}, \alpha_{\theta}\right)>\eta_{k} r_{n} \text { for } r_{n}<\delta_{p} .
$$

For each $n$ the closure of $O_{n}$ contains a point on the boundary of $D$. Let $b_{n}$ be the radius connecting $w_{n}$ to this point and define $b_{n}^{z}$ by requiring $f\left(b_{n}^{z}\right)=b_{n}$. Since $f$ is normal it has no Koebe arcs [4, pp. 262-267]. Thus $b_{n}^{z}$ has an end point $e^{i \theta_{n}}$ on $\{|z|=1\}, e^{i \theta_{n}} \notin E$, and it follows that the image of $b_{n}^{z}$ and the image of the radius at $e^{i \theta_{n}}$ under $f$ determine the same accessible boundary point whose complex coordinate we can now denote by $f\left(e^{i \theta_{n}}\right)$. In addition, since $\operatorname{diam} b_{n} \rightarrow 0$ as $n \rightarrow \infty$, one has by Koebe's lemma [2, p. 31] that $e^{i \theta_{n}} \rightarrow e^{i \theta_{0}}$ as $n \rightarrow \infty$. By choosing a subsequence and relabeling it one can suppose without loss of generality that $\theta_{0}<\theta_{n}<\theta_{0}+\pi / 2$ for all $n$. Setting $E_{n}=\left\{e^{i\left(\theta_{n}-\zeta\right)} \in E: 0<\zeta<\theta_{n}-\theta_{0}\right\}$, we define $\Gamma_{n}$ to be those circular arcs contained in $\{|z|<1\}$ which are orthogonal to $|z|=1$ at $e^{i\left(\theta_{n}+\zeta\right)}$ and $e^{i\left(\theta_{n}-\zeta\right)}$ for some $e^{i\left(\theta_{n}-\zeta\right)} \in E_{n}$. It follows from above that for all $n$,

$$
\lambda\left(\Gamma_{n}\right) \leq \pi / \log k_{n},
$$

where $k_{n}=\sin \left(\theta-\theta_{0}\right) / \sin \left[\left(\theta_{n}-\theta_{0}\right)-m^{*}\left(E_{n}\right)\right]$. Using (2) and the fact that $\sin x \leq x$ for $0 \leq x<\pi / 2$, we have

$$
\sin \left(\theta_{n}-\theta_{0}\right) \leq e^{\pi / \lambda\left(\Gamma_{n}\right)}\left[\left(\theta_{n}-\theta_{0}\right)-m^{*}\left(E_{n}\right)\right],
$$


and hence

$$
\frac{m^{*}\left(E_{n}\right)}{\theta_{n}-\theta_{0}} \leq 1-e^{-\pi / \lambda\left(\Gamma_{n}\right)}\left[\sin \left(\theta_{n}-\theta_{0}\right) /\left(\theta_{n}-\theta_{0}\right)\right] .
$$

From (3) we will be able to conclude that $e^{i \theta_{0}}$ is not a point of outer density providing we establish the existence of a positive number $c$ satisfying $\lambda\left(\Gamma_{n}\right) \geq c$ for all $n$ such that $r_{n}<\delta_{p}$. We will use (1) and the conformal invariance of $\lambda$ to do just this.

Let $\Gamma\left(b_{n}\right)=\left\{\gamma: \gamma\right.$ is a curve in $D$ which joins a point of $b_{n}$ to a point $\left.f\left(e^{i \theta}\right), e^{i \theta} \in E\right\}$ and $\Gamma_{n}^{\prime}=\left\{\gamma^{\prime}: \gamma^{\prime} \subset\{|z|<1\}\right.$ and $f\left(\gamma^{\prime}\right) \in$ $\left.\Gamma\left(b_{n}\right)\right\}$. Then $\lambda\left(\Gamma\left(b_{n}\right)\right)=\lambda\left(\Gamma_{n}^{\prime}\right)$ and since every $\gamma \in \Gamma_{n}$ contains some curve $\gamma^{\prime} \in \Gamma_{n}^{\prime}$ we have by the comparison principle for extremal length that $\lambda\left(\Gamma_{n}^{\prime}\right) \leq \lambda\left(\Gamma_{n}\right)$. Hence to show $e^{i \theta_{0}}$ is not a point of outer density it suffices to find a positive number $c$ satisfying $\lambda\left(\Gamma\left(b_{n}\right)\right) \geq c$ for all $n$ such that $r_{n}<\delta_{p}$.

For each $n$ such that $r_{n}<\delta_{p}$, let $V_{n}=\left\{w \in D\right.$ : $\operatorname{dist}\left(w, b_{n}\right)<$ $\left.\eta_{k} l\left(b_{n}\right)\right\}$, where $\operatorname{dist}\left(w, b_{n}\right)$ denotes the Euclidean distance form the point $w$ to $b_{n}$ and $l()$ denotes the length of the curve. By (1) it follows that for any $\gamma \in \Gamma\left(b_{n}\right)$,

$$
l\left(\gamma \cap V_{n}\right)>\eta_{k} r_{n} .
$$

Defining $\rho_{n}(w)=1$ if $w \in V_{n}$ and 0 elsewhere we have that $\rho_{n}$ is a measurable function, $\int_{\gamma} \rho_{n}|d w| \geq \eta_{k} r_{n}$ for all $\gamma \in \Gamma\left(b_{n}\right)$ and $A\left(\rho_{n}\right)$-the area integral for $V_{n}$ with respect to $\rho_{n}$-satisfies $A\left(\rho_{n}\right) \leq$ $2 \eta_{k} r_{n}^{2}+\pi \eta_{k}^{2} r_{n}^{2}$. Hence for each $n$ such that $r_{n}<\delta_{p}$,

$$
\begin{aligned}
\lambda\left(\Gamma\left(b_{n}\right)\right) & =\sup _{\rho} \frac{L\left(\Gamma\left(b_{n}, \rho\right)\right)^{2}}{A(\rho)} \geq \frac{L\left(\Gamma\left(b_{n}, \rho_{n}\right)\right)^{2}}{A\left(\rho_{n}\right)} \\
& =\frac{\left[\inf _{\gamma \in \Gamma\left(b_{n}\right)} \int_{\gamma} \rho_{n}|d w|\right]^{2}}{A\left(\rho_{n}\right)} \geq \frac{\eta_{k}}{2+\pi \eta_{k}} .
\end{aligned}
$$

Since $\eta_{k}$ is independent of $n$ we choose this last value for $c$.

It follows that $F_{k}^{*}$ and $\bigcup_{k} F_{k}^{*}$ are $D$-conformal null sets and we can conclude that with the exception of a $D$-conformal null set, for each $\alpha \in R^{*}$ and $k, k=1,2, \ldots$, there exists a boundary sequence $\left\{w_{k, n}\right\}$ in $D$ whose corresponding sequence of disks $\left\{O_{k, n}\right\}$ satisfies $l_{D^{*}}\left(O_{k, n}, \alpha\right) \leq \eta_{k} r_{k, n}$ for all $n$. Since $r_{k, n} \rightarrow 0$ as $n \rightarrow \infty$, one has $l_{D^{*}}\left(w_{k, n}, \alpha\right) \rightarrow 0$ as $n \rightarrow \infty$. Using this fact and a relabeling of the sequence we can assume without loss of generality that for each $k$, $l_{D^{*}}\left(w_{k, n}, \alpha\right)<1 / k$ for all $n$. 
If we now consider the rectangular array of points $\left[w_{k, n}\right]$ and form the sequence $\left\{w_{n, n}\right\}$ we arrive at the following result: Except for a $D$-conformal null set of $R^{*}$, for each $\alpha \in R^{*}$ there exists a sequence $\left\{w_{n}\right\} \quad\left(w_{n, n}=w_{n}\right)$ along with its corresponding sequence of disks $\left\{O_{n}\right\}$ which satisfy the following:

(4) $O_{n}$ is contained in $D$ for each $n$,

(5) $l_{D^{*}}\left(w_{n}, \alpha\right) \rightarrow 0$ as $n \rightarrow \infty$,

(6) $l_{D^{*}}\left(O_{n}, \alpha\right) \leq \eta_{n} r_{n}$ for all $n$.

Since $\alpha \in R^{*}$ we have that the Euclidean distance from $O_{n}$ to $w(\alpha), \operatorname{dist}\left(O_{n}, w(\alpha)\right)$, is positive for all $n$. Using (5) there exists a point of the sequence $\left\{w_{n}\right\}$, which we denote by $w_{n_{1}}$ such that $l_{D^{*}}\left(w_{n_{1}}, \alpha\right)<\operatorname{dist}\left(O_{1}, w(\alpha)\right) / 2$. It follows that $\operatorname{dist}\left(w_{n_{1}}, w(\alpha)\right)<$ $\operatorname{dist}\left(O_{1}, w(\alpha)\right) / 2$ and $r_{n_{1}}<\operatorname{dist}\left(O_{1}, w(\alpha)\right) / 2$. Hence the disks $O_{n_{1}}$ and $O_{1}$ are disjoint. Using the fact that $\operatorname{dist}\left(O_{n_{1}}, w(\alpha)\right)$ is positive we repeat the above argument to get a point $w_{n_{2}}, n_{1}<n_{2}$ from the sequence $\left\{w_{n}\right\}$ whose corresponding disk $O_{n_{2}}$ is disjoint from $O_{n_{1}}$ and $O_{1}$. Inductively we are able to define a subsequence $\left\{w_{n_{k}}\right\}$ of $\left\{w_{n}\right\}$ whose corresponding sequence of disks $\left\{O_{n_{k}}\right\}$ satisfy (4), (5), (6) in addition to being pairwise disjoint. By relabeling this sequence one can assume without loss of generality that the $O_{n}$ are pairwise disjoint. Since the sequence

$$
\left\{\nu_{n}=\arg \left(w_{n}-w(\alpha)\right) \bmod (2 \pi), n=1,2, \ldots\right\}
$$

is bounded, it has a subsequence that converges to some $\nu_{0}$ where either $0<\nu_{0}<2 \pi$ or $\nu_{0}=\bmod (2 \pi)$. Thus, we can further assume without loss of generality that the sequence $\left\{w_{n}\right\}$ is such that

$$
\arg \left(w_{n}-w(\alpha)\right) \quad \bmod 2 \pi \rightarrow \nu_{0} \quad \text { as } n \rightarrow \infty .
$$

We shall set $\nu(\alpha)=\nu_{0}$.

For any positive numbers $\delta$ and $\varepsilon, \varepsilon<\pi / 2$, we know from (5) and (7) that for $n$ sufficiently large, $O_{n}$ is contained in $\{|w-w(\alpha)|<\delta\}$ and $O_{n}$ intersects the sector $\Delta=\left\{w(\alpha)+\rho e^{i \phi}: \rho<\delta\right.$ and $\left|\phi-\nu_{0}\right|<$ $\pi / 2-\varepsilon\}$. We show that one can choose $n$ sufficiently large so that the boundary of $O_{n}$ intersects the boundary of $\Delta$ at four distinct points. Let $L_{n}$ denote the ray through $w_{n}$ having terminal point $w(\alpha)$. Let $T_{n}^{\prime}, T_{n}^{\prime \prime}$ denote the two rays tangent to $O_{n}$ having terminal point $w(\alpha)$ and let $\varepsilon_{n}$ denote the measure of the angle formed by $L_{n}$ and $T_{n}^{\prime}$ and $L_{n}$ and $T_{n}^{\prime \prime}$. See Figure 1. If $d_{n}$ denotes the Euclidean distance from $O_{n}$ to $w(\alpha)$, we have that

$$
\sin \varepsilon_{n}=\frac{r_{n}}{r_{n}+d_{n}}>\frac{r_{n}}{r_{n}+l_{D^{*}}\left(O_{n}, \alpha\right)}=\frac{1}{1+l_{D^{*}}\left(O_{n}, \alpha\right) / r_{n}} .
$$




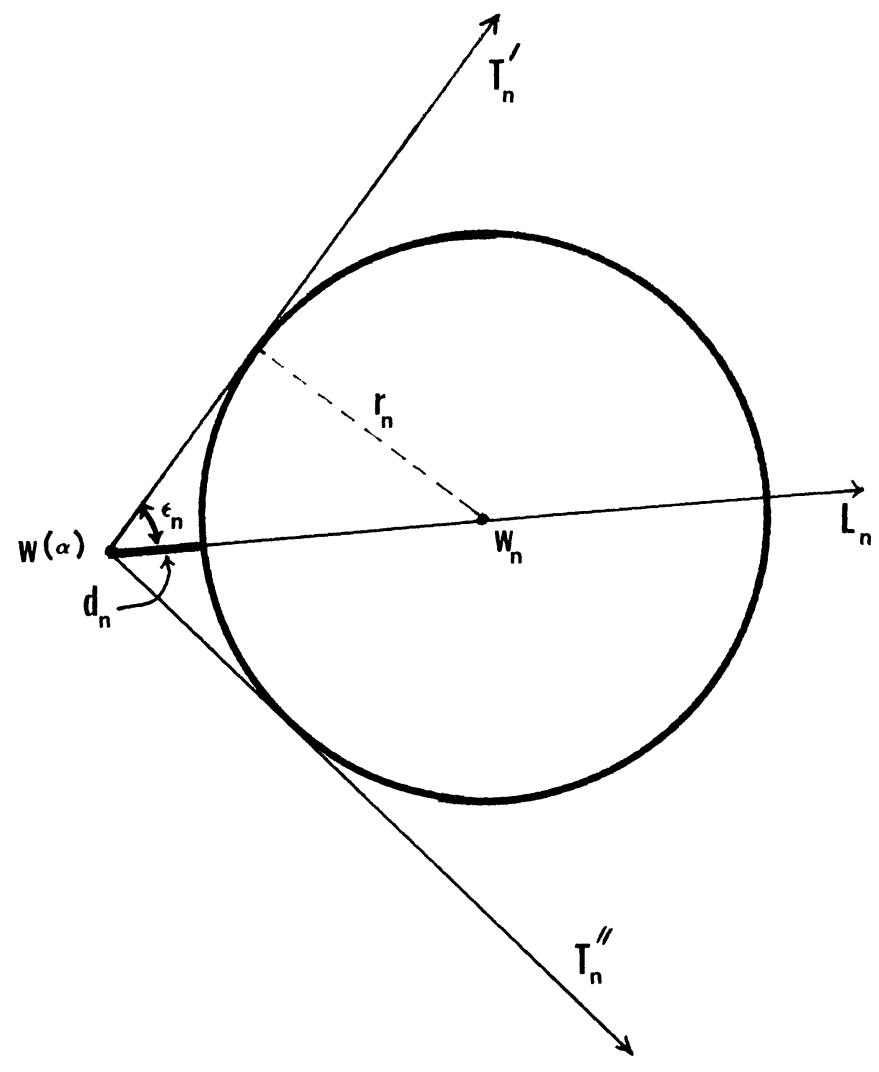

FIGURE 1

Using (6) we see that as $n \rightarrow \infty, \sin \varepsilon_{n} \rightarrow 1$ and so $\varepsilon_{n} \rightarrow \pi / 2$. If we choose $n$ sufficiently large so that

$$
\varepsilon_{n}>\pi / 2-\varepsilon / 2 \text { and }\left|\arg L_{n}-\nu_{0}\right|<\varepsilon / 4
$$

then our claim follows. Note that one pair of the four points lies on the segment $w(\alpha)+\rho e^{i\left(\nu_{0}+(\pi / 2-\varepsilon)\right)}: 0 \leq \rho \leq \delta$ and the other pair lies on the segment $w(\alpha)+\rho e^{i\left(\nu_{0}-(\pi / 2-\varepsilon)\right)}: 0 \leq \rho \leq \delta$. We choose the pair whose Euclidean distance from one another is the smaller and denote their distance from $w(\alpha)$ by $\delta_{n}^{\prime}$ and $\delta_{n}^{\prime \prime}$ where $\delta_{n}^{\prime}<\delta_{n}^{\prime \prime}$. Doing this for each $n, n$ sufficiently large, defines two sequences $\left\{\delta_{n}^{\prime}\right\},\left\{\delta_{n}^{\prime \prime}\right\}$ such that

$$
A\left(\alpha, \nu_{0}, \delta_{n}^{\prime}, \delta_{n}^{\prime \prime}, \pi / 2-\varepsilon\right) \subset O_{n} \subset D \text { for all } n
$$

Letting $\tau_{n}=\left[\arg \left(w_{n}-w(\alpha)\right) \bmod 2 \pi-\nu_{0}\right]$ and referring to Figure 


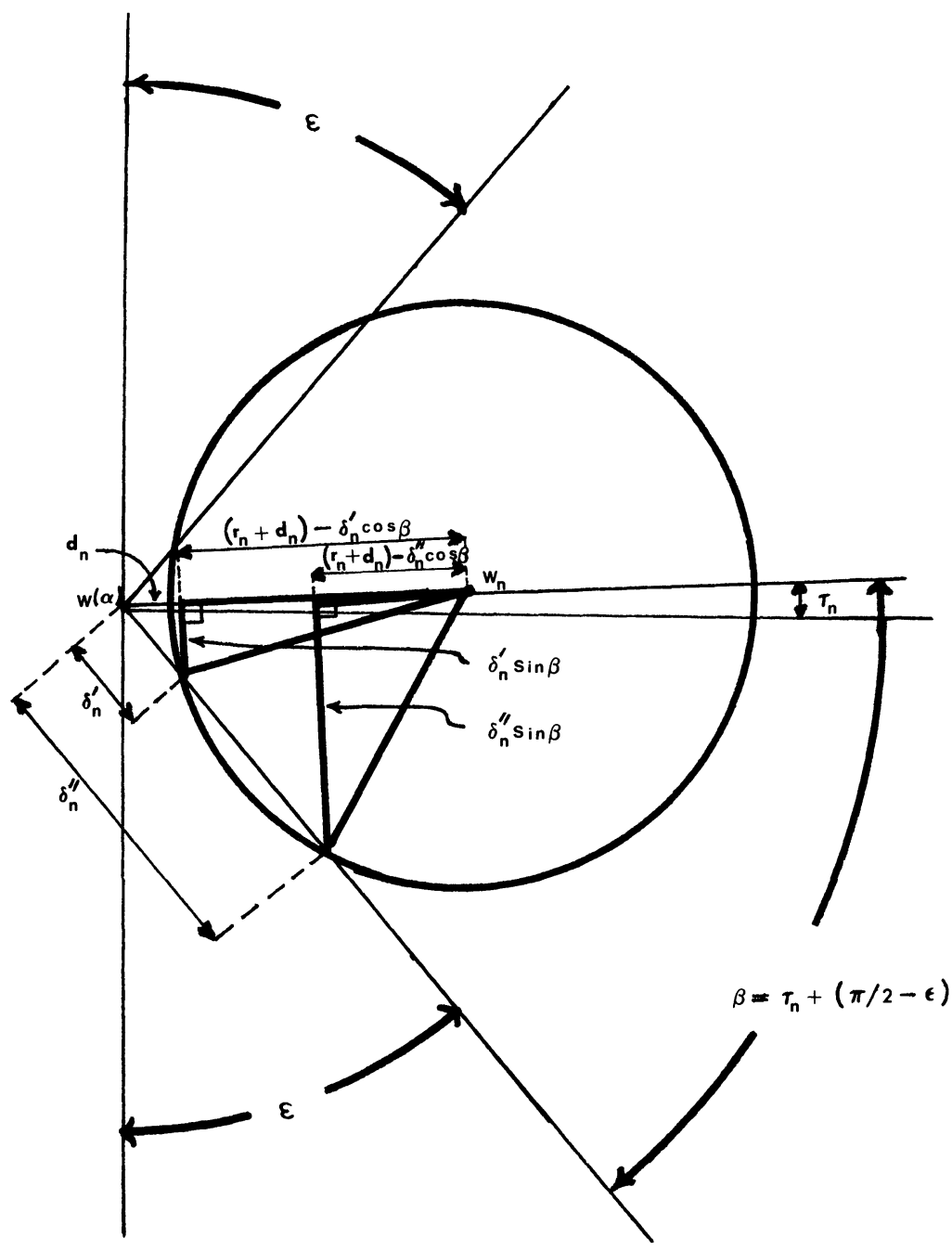

FIGURE 2

2 we have that

$$
\begin{aligned}
& {\left[\delta_{n}^{\prime} \sin \left(\tau_{n}+(\pi / 2-\varepsilon)\right)\right]^{2}+\left[\left(r_{n}+d_{n}\right)-\delta_{n}^{\prime} \cos \left(\tau_{n}+(\pi / 2-\varepsilon)\right)\right]^{2}=r_{n}^{2}} \\
& {\left[\delta_{n}^{\prime \prime} \sin \left(\tau_{n}+(\pi / 2-\varepsilon)\right)\right]^{2}+\left[\left(r_{n}+d_{n}\right)-\delta_{n}^{\prime \prime} \cos \left(\tau_{n}+(\pi / 2-\varepsilon)\right)\right]^{2}=r_{n}^{2}}
\end{aligned}
$$

Upon solving for $\delta_{n}^{\prime}$ and $\delta_{n}^{\prime \prime}$ we have that

$$
\frac{\delta_{n}^{\prime \prime}-\delta_{n}^{\prime}}{r_{n}}=2 \frac{r_{n}+d_{n}}{r_{n}} \sqrt{\cos \left(\tau_{n}+(\pi / 2-\varepsilon)\right)^{2}+r_{n}^{2} /\left(r_{n}+d_{n}\right)^{2}-1}
$$




$$
\begin{aligned}
& \frac{\delta_{n}^{\prime \prime}-\delta_{n}^{\prime}}{\delta_{n}^{\prime \prime}} \\
& \quad=\frac{2 \sqrt{\cos \left(\tau_{n}+(\pi / 2-\varepsilon)\right)^{2}+r_{n}^{2} /\left(r_{n}+d_{n}\right)^{2}-1}}{\cos \left(\tau_{n}+(\pi / 2-\varepsilon)\right)+\sqrt{\cos \left(\tau_{n}+(\pi / 2-\varepsilon)\right)^{2}+r_{n}^{2} /\left(r_{n}+d_{n}\right)^{2}-1}} .
\end{aligned}
$$

Hence

$$
\lim _{n \rightarrow \infty} \frac{\delta_{n}^{\prime \prime}-\delta_{n}^{\prime}}{r_{n}}=2 \cos (\pi / 2-\varepsilon), \quad \lim _{n \rightarrow \infty} \frac{\delta_{n}^{\prime \prime}-\delta_{n}^{\prime}}{\delta_{n}^{\prime \prime}}=1,
$$

and the lemma is proven.

Proof of Theorem. Suppose to the contrary that $W^{*}$ is not $D$ conformal null. By the lemma, with the exception of a $D$-conformal null set of $W^{*}$ we know that for each $\alpha \in W^{*}$ there exists $\nu(\alpha)$, $0 \leq \nu(\alpha) \leq 2 \pi$, such that for $\varepsilon=\pi / 12$ there exist sequences $\left\{\delta_{n}^{\prime}\right\}$, $\left\{\delta_{n}^{\prime \prime}\right\}$ such that

$$
A\left(\alpha, \nu(\alpha), \delta_{n}^{\prime}, \delta_{n}^{\prime \prime}, 5 \pi / 12\right) \subset D \text { for all } n
$$

and

$$
\lim _{n \rightarrow \infty} \frac{\delta_{n}^{\prime \prime}-\delta_{n}^{\prime}}{\delta_{n}^{\prime \prime}}=1
$$

Also, from the definition of $W^{*}$, for each $\alpha \in W^{*}$ there exists a $k, k=1,2,3$, such that $L_{\nu_{k}}$ passes through the regions $A\left(\alpha, \nu(\alpha), \delta_{n}^{\prime}, \delta_{n}^{\prime \prime}, 5 \pi / 12\right)$ and $u\left(\nu_{k}\right)<1$. However,

$$
\frac{m\left(\left(L_{\nu_{k}} \cap D\right) \cap\left(w(\alpha), w(\alpha)+\delta_{n}^{\prime \prime} e^{i \nu_{k}}\right)\right)}{\delta_{n}^{\prime \prime}}>\frac{\delta_{n}^{\prime \prime}-\delta_{n}^{\prime}}{\delta_{n}^{\prime \prime}}
$$

and this implies that $u\left(\nu_{k}\right)=1$. This is a contradiction. Thus $W^{*}$ must be a $D$-conformal null set.

\section{REFERENCES}

[1] T. E. Gerasch, On the accessibility of the boundary of a simply connected domain, Michigan Math. J., 33 (1986), 201-207.

[2] G. M. Goluzin, Geometric Theory of Functions of a Complex Variable, vol. 26, Transl. Math. Monographs, Amer. Math. Soc., Providence, R.I., 1969.

[3] J. E. McMillan, Boundary behavior of a conformal mapping, Acta Math., 123 (1969), 43-67.

[4] Ch. Pommerenke, Univalent Functions, Vandenhoeck and Ruprecht, Gottingen, 1975.

Received August 14, 1989 and in revised form February 5, 1991. 



\section{PACIFIC JOURNAL OF MATHEMATICS EDITORS}

V. S. VARADARAJAN

(Managing Editor)

University of California

Los Angeles, CA 90024-1555-05

Herbert Clemens

University of Utah

Salt Lake City, UT 84112

F. Michael Christ

University of California

Los Angeles, CA 90024-1555

THOMAS ENRIGHT

University of California, San Diego

La Jolla, CA 92093

\section{C. MOORE}

University of California

Berkeley, CA 94720

MARTIN SCHARLEMANN

University of California

Santa Barbara, CA 93106

HAROLD STARK

University of California, San Diego

La Jolla, CA 92093

STEVEN KERCKHOFF

Stanford University

Stanford, CA 94305

ASSOCIATE EDITORS

R. ARENS

E. F. BECKENBACH (1906-1982)

B. H. NeumanN

F. WOLF

(1904-1989)

K. YosHIDA

SUPPORTING INSTITUTIONS

UNIVERSITY OF ARIZONA

UNIVERSITY OF OREGON

UNIVERSITY OF BRITISH COLUMBIA

UNIVERSITY OF SOUTHERN CALIFORNIA

CALIFORNIA INSTITUTE OF TECHNOLOGY

UNIVERSITY OF CALIFORNIA

MONTANA STATE UNIVERSITY

STANFORD UNIVERSITY

UNIVERSITY OF NEVADA, RENO

UNIVERSITY OF HAWAII

NEW MEXICO STATE UNIVERSITY

OREGON STATE UNIVERSITY

UNIVERSITY OF UTAH

WASHINGTON STATE UNIVERSITY

UNIVERSITY OF WASHINGTON 


\section{Pacific Journal of Mathematics}

\section{Vol. 153, No. $1 \quad$ March, 1992}

Patrick Robert Ahern and Carmen Cascante, Exceptional sets for Poisson integrals of potentials on the unit sphere in $\mathbf{C}^{n}, p \leq 1 \ldots \ldots \ldots \ldots \ldots$

David Peter Blecher, The standard dual of an operator space ........... 15

Patrick Gilmer, Real algebraic curves and link cobordism .............. 31

Simon M. Goberstein, On orthodox semigroups determined by their

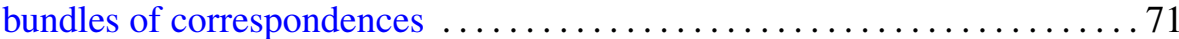

John Kalliongis and Darryl John McCullough, Homeotopy groups of irreducible 3-manifolds which may contain two-sided projective planes .......................................... 85

Yuji Konishi, Masaru Nagisa and Yasuo Watatani, Some remarks on actions of compact matrix quantum groups on $C^{*}$-algebras $\ldots \ldots \ldots \ldots 119$

Guojun Liao and Luen-Fai Tam, On the heat equation for harmonic maps from noncompact manifolds ........................... 129

John Marafino, Boundary behavior of a conformal mapping . .......... 147

Ji Min, A remark on the symmetry of solutions to nonlinear elliptic

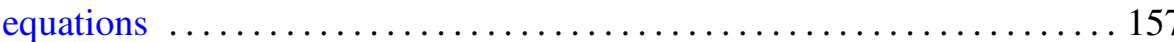

Paul Nevai and Walter Van Assche, Compact perturbations of orthogonal

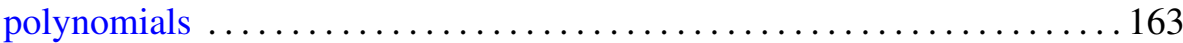

Kyril Tintarev, Level set maxima and quasilinear elliptic problems ....... 185 